\title{
An update on the maize zein-gene family in the post-genomics era
}

\author{
Nasr Ullah Khan ${ }^{1}$, Mohamed Sheteiwy ${ }^{1,2}$, Ning Lihua', Muhammad Mohib Ullah Khan ${ }^{3}$ and Zhao Han ${ }^{1 *}$
}

\begin{abstract}
Maize (Zea mays) is a cereal crop of global food importance. However, the deficiency of essential amino acids, more importantly lysine, methionine and tryptophan, in the major seed storage zein proteins makes corn nutritionally of low value for human consumption. The idea of improving maize nutritional value prompted the search for maize natural mutants harboring low zein contents and higher amount of lysine. These studies resulted in the identification of more than dozens of maize opaque mutants in the previous few decades, 02 mutant being the most extensively studied one. However, the high lysine contents but soft kernel texture and chalky endosperm halted the widespread application and commercial success of maize opaque mutants, which ultimately paved the way for the development of Quality Protein Maize (QPM) by modifying the soft endosperm of o2 mutant into lysine-rich hard endosperm. The previous few decades have witnessed a marked progress in maize zein research. It includes elucidation of molecular mechanism underlying the role of different zein genes in seed endosperm development by cloning different components of zein family, exploring the general organization, function and evolution of zein family members within maize species and among other cereals, and elucidating the cis- and trans-regulatory elements modulating the regulation of different molecular players of maize seed endosperm development. The current advances in high quality reference genomes of maize lines B73 and Mo17 plus the completion of ongoing pan genome sequencing projects of more maize lines with NGS technologies are expected to revolutionize maize zein gene research in near future. This review highlights the recent advances in QPM development and its practical application in the post genomic era, genomic and physical composition and evolution of zein family, and expression, regulation and downstream role of zein genes in endosperm development. Moreover, recent genomic tools and methods developed for functional validation of maize zein genes are also discussed.
\end{abstract}

Keywords: Zein, Opaque mutants, QPM, NGS

\section{Introduction}

Rich in endosperm storage proteins and starch, maize is the most important cereal crop in the world; both as food and feed. Maize endosperm zein protein belongs to prolamines which are the most abundant type of proteins stored in cereals seeds like wheat, maize, sorghum, rice and barley etc. (Holding 2014). Maize and sorghum are the repertoires of essential amino acids and biological nitrogen, thus the functional study of their prolamines is important from environmental and economic point of view. Zein belongs to the most abundant type of proteins, representing $70 \%$ of the total $10 \%$ proteins

\footnotetext{
* Correspondence: zhaohan@jaas.ac.cn

${ }^{1}$ Institute of Crop Germplasm and Biotechnology, Provincial Key Laboratory of Agrobiology, Jiangsu Academy of Agricultural Sciences, Nanjing 210014, China

Full list of author information is available at the end of the article
}

stored in maize seed (Flint-Garcia, Bodnar \& Scott 2009; Wu \& Messing 2014). However, similar to other cereals, maize is of low nutritional value as its zein proteins are deficient of tryptophan and lysine, which are the two most important essential amino acids. The amount of tryptophan and lysine $(0.25-0.50 \%$ and $1.5-2.5 \%$, respectively) in maize seed is much lesser than the minimal level (1.1 and 5\% for tryptophan and lysine, respectively) required in human diet (Ren et al. 2018). Thus, the accumulation of tryptophan and lysinedeficient zein in maize kernel leads to poor quality of grain proteins. Long ago, maize breeders have identified maize opaque2 (o2) mutant which exhibited as much as twice tryptophan and lysine contents than the wild type and showed great promise for livestock and human consumption (Osborne \& Mendel 1914). Maize o2 mutant

(c) The Author(s). 2019 Open Access This article is distributed under the terms of the Creative Commons Attribution 4.0 International License (http://creativecommons.org/licenses/by/4.0/), which permits unrestricted use, distribution, and 
was followed by the identification of more than dozens of maize opaque or floury mutants. Most notable features of such mutants include soft and chalky seeds with opaque endosperm, sterility and abnormal kernel development. The relatively higher nutritional value (elevated lysine contents) and chalky texture of maize opaque endosperm mutants were selected as superior traits by corn breeders to clone and characterize the genes responsible for opaque endosperm. More than dozens of genes underlying well-known opaque mutants have been cloned and characterized to decipher the molecular basis of the opaque endosperm phenotype (Table 1). The genes or loci controlling the zein protein and ultimately opaque or floury phenotypes falls into three categories. The first group includes the genes which encode zein proteins $(M c$, $D e-B 30, F L 2$ and $F L 4$ ) or non-zein proteins as integral part of protein bodies (FL1, O1 and 010), the second one encodes enzymes regulating endosperm metabolism $(\mathrm{O} 5$, $O 6$ and 07$)$ and the third group includes transcriptional regulatory genes (O2, O11 and FL3) (Zhang et al. 2018).

In maize endosperm, zein proteins reside in protein bodies (PBs) inside endoplasmic reticulum (ER). Considerable evidences suggested that the proper accumulation and packaging of zein and non-zein proteins into the PBs have a central role in maize endosperm development during maize kernel maturation and ultimately influence kernel texture, functionality and protein quality (Guo et al. 2013; Holding et al. 2007; Kim et al. 2002; Zhang et al. 2018). Different types of mutations lead to maize opaque or floury endosperm phenotype. In majority of well-known maize opaque or floury mutants, the mutation affects the normal metabolism, development, number and physical shape of protein bodies, thereby, producing an opaque or chalky endosperm. For instance, a point mutation in signal peptide cleavage site in $19-\mathrm{kD}$ $\alpha$-zein in $f l 4$ and $D e-B 30$ mutants changes accumulation of zein and produce $\mathrm{PBs}$ with irregular structure (Kim et al. 2004; Wang, Qi et al. 2014). Contrary to $f l 4$ and $D e-B 30$, a frame shift mutation in the exon of $16-\mathrm{kDa} \gamma-$ zein gene leads to $M c$ mutant with miss-shaped protein bodies (Kim et al. 2006). In case of $o 1$ mutant, mutation in the myosin XI motor-like protein encoding $\mathrm{O} 1$ gene generates small and somehow miss-shaped PBs as compared to wild type, without affecting the synthesis of zein proteins (Wang et al. 2012). On the contrary, mutants also exhibit opaque phenotype when mutation happens in genes that code for metabolic enzymes, thus ultimately change the zein deposition and formation of PBs. For example, mutation in Monogalactosyldiacylglycerol synthase (MGD1) in o5 mutant dysfunctions chloroplast and amyloplast, and additionally affects the structure and physical appearance of starch particles inside PBs (Myers et al. 2011). On the other hand, maize 07 mutant known for its high lysine contents was shown to be regulated by $Z m O C D 1$, which catalyzes the product of $O 7$ (commonly oxalate) and ultimately alter the kernel metabolic processes, endosperm formation and nutritional status (Yang, Fu, Ji, Huang \& Wu 2018). Moreover, mutation in transcriptional regulatory genes also leads to opaque phenotype. Opaque2 (O2) is the most extensively studied and well known mutant where the opaque endosperm arises from loss of function mutation in the $\mathrm{O} 2$ transcriptional factor (Zhang, Zheng, Yang, Messing \& Wu 2016). Despite of increased lysine contents and enhanced nutritional quality, the widespread adoption of 02 mutant was halted by its chalky

Table 1 List of maize opaque endosperm mutants identified and characterized so far. Modified from Zhang, Zhan \& Yadegari (2018)

\begin{tabular}{|c|c|c|c|c|}
\hline Mutant & Locus & Gene name & Protein type & References \\
\hline 01 & Zm00001d052110 & - & Myosin XI motor & Wang et al. (2012) \\
\hline 02 & Zm00001d018971 & - & bZIP TF & $\begin{array}{l}\text { Schmidt, Ketudat, Aukerman } \\
\text { \& Hoschek (1992) }\end{array}$ \\
\hline 05 & Zm00001d020537 & MGD1 & Monogalactosyldiacylglycerol synthase & Myers et al. (2011) \\
\hline 06 & Zm00001d010056 & PRO1 & D1-pyrroline-5-carboxylate synthetase & Wang et al. (2014) \\
\hline 07 & Zm00001d026649 & $A A E 3$ & Acyl-CoA synthetase & Miclaus, Xu \& Messing (2011) \\
\hline 010 & Zm00001d033654 & - & Cereal-specifc non-zein PB protein & Yao et al. (2016) \\
\hline 011 & Zm00001d003677 & - & bHLH TF & Feng et al. (2018) \\
\hline$f / 1$ & Zm00001d003398 & - & Non-zein PB protein & Holding et al. (2007) \\
\hline$f 12$ & Zm00001d049243 & - & 22-kD a-zein & Coleman et al. 1997 \\
\hline $\mathrm{fl3}$ & Zm00001d009292 & - & PLATZ TF & Li et al. (2017) \\
\hline$f / 4$ & Zm00001d048851 & - & 19-kD a-zeins & Wang et al. (2014) \\
\hline De-B30 & Zm00001d019158 & - & 19-kD a-zeins & Kim et al. (2004) \\
\hline MC & Zm00001d005793 & - & 16-kD y-zein & Kim et al. (2006) \\
\hline mto140 & Zm00001d014734 & AroDH-1 & Arogenate dehydrogenase & Holding et al. (2010) \\
\hline
\end{tabular}

De-B30 defective endosperm-B30, $f$ floury, Mc Mucronate, o opaque, PB protein body 
endosperm, which is easily damaged by fungi, pests and mechanical shearing. The world-wide breeding efforts produced nutritionally superior maize cultivars with hard kernels and higher lysine contents, commonly known as quality protein maize (QPM).

\section{QPM; genetic and theoretical basis and practical significance}

Maize breeders have long ago focused on improving the kernel hardness as a trait of agronomic importance which not only provides resistance to damage caused by insect and fungal attack but also protects the seeds from physical breakage during harvesting, handling and storage (Wu, Holding \& Messing 2010). The relative amount of peripheral hard (vitreous) and central soft (opaque) parts determines the maize kernel texture. Kernel vitreousness and zein accumulation are directly related. Accumulation of high zein and low lysine reflects a small central opaque and wider peripheral vitreous regions, while low zein and high lysine contents determines the wider opaque region which extends from center to the periphery in the kernels of $o 2$ mutant (Holding 2014). The core objective of developing QPM was to modify the $o 2$ mutant chalky endosperm, by utilizing QTLs known as o2 modifiers $(\mathrm{Opm})$, thereby restoring kernel vitreousness and hardness while retaining high lysine and low $\alpha$-zein contents. Thus, QPM line is reflective of $o 2$ mutant and ordinary maize in terms of elevated lysine level and high yield, respectively (Fig. 1). However, the technical issues involved in integrating such wide spread $\mathrm{Opm}$ loci in QPM lines slowed down the process of development and world-wide applications of the newly developed QPM varieties. Moreover, such technical complexity of developing QPM lines was further aggravated by an uneasy assessment of amino acid composition, obscure identities plus uneven distribution of Opm loci and problems in introducing an $o 2$

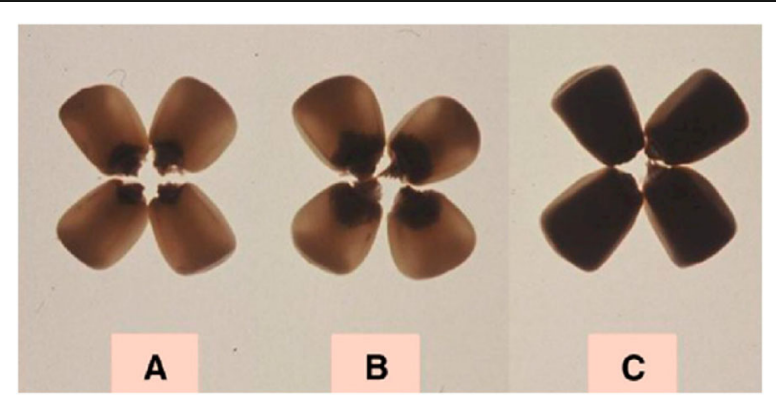

Fig. 1 Back-lit maize kernels illustrating the phenotypic differences of opaque2 mutation. $\mathbf{a}, \mathbf{b}$ and $\mathbf{c}$ represent common maize, QPM and opaque2 maize, respectively. Taken from Nuss and Tanumihardjo (2011) homozygous locus (Babu, Agrawal, Saha \& Gupta 2015; Holding et al. 2008).

Breeding efforts in the past couples of decades have identified several $o 2$ modifiers and shed light on their role in modifying QPM endosperm; however, both the mechanism and genetic components controlling endosperm modification remained complex and challenging. Different genetic mapping studies have identified seven o2 modifiers with major and minor phenotypic effects on kernel endosperm development, and mainly distributed over six chromosomes. Six of them have smaller phenotypic effects while one major effect locus, known as Opm1, is located near $27 \mathrm{kDa} \gamma$-zein locus (Babu et al. 2015; Holding et al. 2008). Few key studies have demonstrated that QPM lines accumulate as much as twice the amount of $27 \mathrm{kDa} \gamma$-zein relative to $o 2$ mutant and wild type maize. Though, a strong correlation has been determined for the opaque endosperm phenotype and the accumulation of $27-\mathrm{kD} \gamma$-zein, the mechanism underlying the elevation of the corresponding zein protein in QPM lines is poorly understood (Geetha, Lending, Lopes, Wallace \& Larkins 1991; Holding 2014; Lopes \& Larkins 1991; Wu et al. 2010). When the $F_{2}$ population constructed from the cross of QPM inbred and $o 2 \mathrm{mu}-$ tant was investigated, a positive association of $27 \mathrm{kDa} \gamma$ zein with opaque trait was revealed (Holding et al. 2011). Recent positional cloning of $27-\mathrm{kD} \gamma$-zein gene validated that the characteristics increase in the $27-\mathrm{kD}$ $\gamma$-zein protein is the outcome of recent evolutionary event, characterized by duplication of the respective locus in modern day maize (Liu et al. 2016). PBs acts as the storehouses of zein proteins. It accumulates a tremendous amount of $\gamma$ zein proteins which are aggregated inside the QPM endosperm to give it a tough and transparent appearance similar to one in the wild type maize. Though, $\gamma$-zein role is pivotal in modifying the QPM endosperm, how solely this protein contributes to the modification process has yet to be determined ( $\mathrm{Wu}$ et al. 2010). The $16-\mathrm{kDa}$ and $15-\mathrm{kDa} \gamma$ - and $\beta$ - zeins in combination with $27-\mathrm{kDa} \gamma$ have been shown to govern the process of initiating and stabilizing the development of PBs. Targeting the $\gamma$-zeins, by RNA interference strategy in QPM lines, exhibited some clear phenotypic changes in PBs, such as decrease in their number, missshaped and clumpy appearance with ultimate soft endosperm like phenotype (Yuan, Dou, Kianian, Zhang \& Holding 2014).

The global population is expected to rise up to about 8.5 billion people by the end of 2030. Such an explosive population requires a double food production in coming years (Wheeler \& von Braun 2013) in order to compensate the food security problems. Enhancing the nutritional quality of staple crops is one strategy to meet effectively the global food demand. QPM varieties were 
developed in order to address the malnutrition problems in the developing countries where people, especially the infants and children, suffer severe protein deficiency. The introduction and utilization of QPM varieties worldwide significantly benefited the people in underdeveloped nations and alleviated the malnutrition problems, especially in Africa (Nuss \& Tanumihardjo 2011). Maize is central to African diet where it is used as a staple food and major nutritional source. Maize is a common source of both micro and macro nutrients mandatory in human's balanced diet, but it lacks some appropriate amounts of essential amino acids, such as lysine and tryptophan. The deficient intake of lysine and tryptophan diet, on daily basis, results in pandemic protein malnutrition which ultimately causes some critical life threatening disorders. The most common disorders are gastroenteritis and tuberculosis (TB) (Rolfes, Pinna \& Whitney 2009). Such malnutrition highly affects the growth of infants when shifted from breast feeding to regular diet and leads to growth retardation in early developmental phase of a child (Planta, Xiang, Leustek \& Messing 2017). The daily consumption of lysine and tryptophan-rich QPM diet, contrary to normal maize, has greatly improved the health of children prone to severe malnutrition (Akalu, Taffesse, Gunaratna \& De Groote 2010). A study has found promising outcomes in Mexico, India and three African countries Ghana, Nicaragua and Ethiopia in which children were shifted from normal maize diet to QPM diet (an average 12\% increase in height and $9 \%$ increase in weight) (Gunaratna, De Groote, Nestel, Pixley \& McCabe 2010). As a promising alternative of the normal maize, yet
QPM varieties have faced numerous challenges in the context of fear from the general public to consider QPM as GM food, commercialization issues and unavailability of suitable market (Nuss \& Tanumihardjo 2011).

\section{The physical and genomic composition and evolution}

Maize seed proteins are broadly categorized into four different types of classes on the basis of their solubility in alcohol and other solvents. The first three classes comprise of glutamines, albumins and globulins, while the fourth class includes zeins (also known as prolamines). Zein proteins constitute $>60 \%$ of the total stored proteins in endosperm of maize kernel (Fig. 2). On the basis of their resolution on SDS gel and physical composition, zein proteins are classified as group of 4 sub-families. These four sub-families include $\alpha$ (22- and 19- kDa), $\beta$ (15-kDa), $\gamma(50-, 27-$, and $16-\mathrm{kDa})$, and $\delta$ (18- and 10-kDa) (Feng et al. 2009; Holding 2014; Song, Llaca, Linton \& Messing 2001; Song \& Messing 2002). Based on the amino acid composition and homology, zeins are broadly categorized into two groups; zein 1 and zein2. Zein1 group (z1) includes two $\alpha$-zeins proteins (19- and $22-\mathrm{kDa}$ ) and zein2 group (z2) contains six different $\delta, \beta$ and $\gamma$ zeins (10-, 18-kDa $\delta$-zein; $15-\mathrm{kDa}$ $\beta$-zein; and 16-, 27-, 50-kDa $\gamma$-zeins) (Xu \& Messing $2009 \mathrm{~b})$. The genes encoding $\delta$-, $\beta$ - and $\gamma$-zeins from z2 group are distributed on different chromosomes in maize genome. These three types of zein proteins are encoded by genes $z 2 \delta 10, z 2 \delta 18, z 2 \beta 15, z 2 \gamma 16, z 2 \gamma 27$ and $z 2 \gamma 50$, respectively. In contrast, $\alpha$-zein from $\mathrm{z} 1$ group constitutes a multi-copy gene family in maize

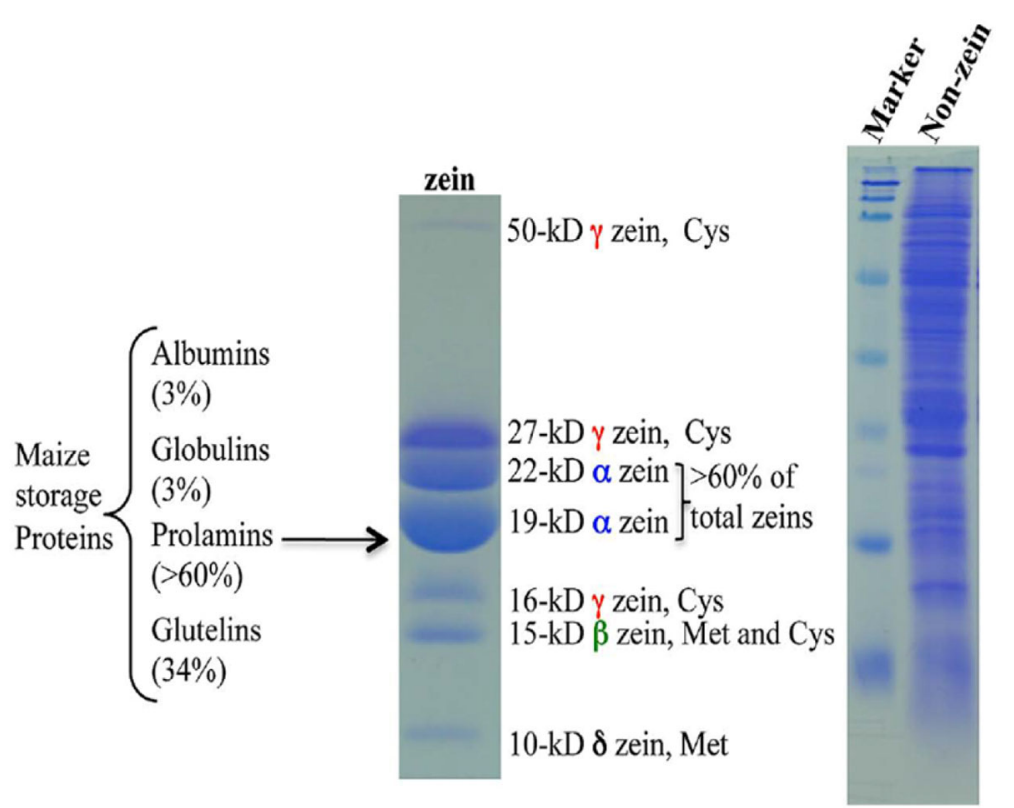

Fig. 2 Maize storage proteins. Prolamin proteins in maize are called zeins and the others are all classified as non-zeins. Adapted from Wu et al. (2012) 
genome. The reference genome of B73 maize inbred line contains more than $44 \alpha$-zeins genes, grouped as four types of sub-gene families $(z 1 A, z 1 B, z 1 C$ and $z 1 D)$ distributed over different chromosomes. The sub-families $z 1 A, z 1 B$ and $z 1 C$ encode 19 - $\mathrm{kDa} \alpha$-zein proteins, while sub-family $z 1 C$ encodes $22-\mathrm{kDa} \alpha-$ zein (Feng et al. 2009). The multi-genic $\alpha$-zein family has undergone extensive gene duplication events and has shown variation in the expression level and the number of copies available per genome in different maize genotypes (Holding 2014). The members of $\alpha$ zein family are distributed on various chromosomes in maize, but more abundantly located on chromosome 4.

Maize was domesticated almost nine thousand years ago from wild grass, teosinte, in Mexico. Since then, maize genome has undergone an extensive evolutionary changes in the form of chromosomal and gene duplication, genes loss and gene imprinting. All these three events that happened in multi-gene families, for instance zein family in maize, have contributed to the genomic organization of present day maize, before and after self-hybridization of diploid genomes of maize. Maize zein-family represents one of the classical examples of evolutionary studies, which helps to understand the phenomenon of single gene-family evolution into multi-copy gene family and how such copies are differentially regulated in different plant species. Several studies have analyzed zein family evolution by syntenic alignments of maize zein gene sequences with other cereals like sorghum, rice, wheat and barley by taking advantage of the good co-linearity of cereals genomes at prolamines loci (Xu \& Messing 2009a, b). Based on evolutionary studies, it has been shown that both sorghum and maize shared a common progenitor with rice, where later both split from rice and formed a separate clade about more than 40 million years ago. Thus, maize zein-gene family can be effectively used to trace all the evolutionary events that happened in the form of duplication, gene loss and gene copying before and after the origin of zein family in Poaceae (Kellogg 2001; Swigonova et al. 2004). Both $\alpha$ - and $\gamma$-zein have been extensively amplified during the course of evolution (Xu \& Messing $2009 a, b)$. Among all zein genes, $\alpha$-zein family is the most recently originated during the phylogenetic split of Panicoideae from Pooideae, about 26 million years ago. The former includes millet, sorghum and maize while the later include oat, barley and wheat (Xu \& Messing 2009b). Interestingly, the first copying event of $\alpha$-zein genes that happened more than 11 million years ago, before the split of maize and sorghum progenitors, led to the formation of a new prolamine z1 locus, containing two zeins of $19 \mathrm{kDa}$ and $20 \mathrm{kDa}$ sizes, respectively. Later on, additional genes re-arrangement and duplication plus gene loss and gain governed by mutation events further shaped the zein-gene family both in maize and sorghum. Maize is the allotetraploid outcome of hybridization event that happened between its two diploid progenitors several million years ago. During the process of evolution, only one of the progenitors retained the new $\alpha$-zein locus, which later on remained un-duplicated in modern day maize (Miclaus et al. 2011). The non-functional gene copies that happened to be damaged by mutations during evolution showed stability for long time. Several studies have shown that such truncated gene copies or those with premature stop codons were still able to express and accumulate their transcripts at minimal level (Liu \& Rubenstein 1993; Song \& Messing 2003; Van Hoof \& Green 1996). It is worthy to know that plants might exploit such copying events as a suitable strategy to adapt themselves to the variations that happens in the ever-changing environment they face. This might be one possible explanation for the multi-copy origin of $\alpha$-zein family, where a common gene copy in the progenitor of maize, initially located at chromosome 1, finally become multi-gene family with more than 40 gene members in B73 reference genome. The members of $\alpha$-zein family have also been shown to vary across different maize backgrounds. For instance, in order to investigate the haplotype-specific and nonspecific variation in the physical region spanning $\alpha$-zein family in three inbreds B73, W22 and BSSS53, it was found that the members of different haplotypes show variation in the gene sequence and number of gene copies per genome. Additionally, variation in expression level among all the three inbred backgrounds was observed (Fig. 3) (Dong et al. 2016; Miclaus et al. 2011). Variation in sequence and gene copy number is the attributes of enormous transposition events that happened after the hybridization of diploid genomes of two maize progenitors, while variation in expression level of zein family members across different background are related to imprinting and epigenetic regulation (Miclaus et al. 2011). Besides $\alpha$-zein family, 27- kDa- $\gamma$ zein gene also showed haplotype and copy number variation on chromosome 7 (Das, Levi-Minzi, Koury, Benner \& Messing 1990). Moreover, the presence/absence polymorphism in $\delta, \gamma$ and $\beta$ genes in maize and sorghum has shown that maize genome has retained the newly duplicated genes copies and lost the older copies of $\beta, \gamma$ and $\delta$ genes that existed in the maize progenitor before allotetraploidization (Xu \& Messing 2009b).

\section{The expression and regulation of zein genes}

For decades, the transcriptional regulation of zein genes has attracted the interest of breeders and researchers due to enormous complexity and high expression rate, besides their tissue- (endosperm) specific expression. Among zein genes, the multi copy status makes 19- and $22-\mathrm{kD} \alpha$-zeins as the most abundant types of zein 


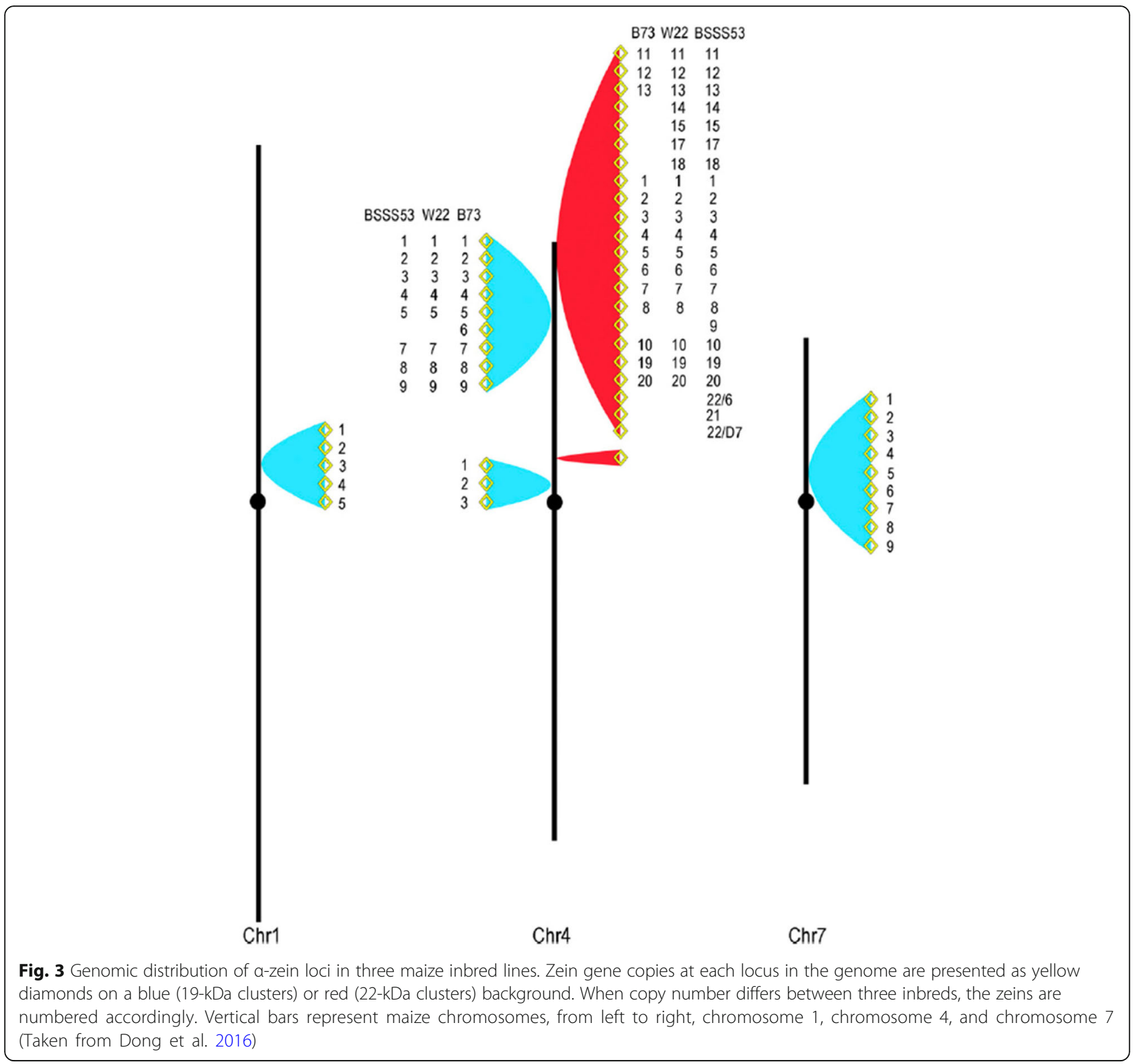

proteins. More than $60 \%$ of maize zein proteins belong to these two types of $\alpha$-zeins. Moreover, majority of the $\alpha$-zein genes with intact functional copies are considered among the top one hundred abundantly expressed genes in maize seed endosperm (Chen et al. 2014). The existence of $\alpha$-zeins as the most prevalent class of zein proteins in maize endosperm is their active and strong expression. The expression and regulation of zein genes involve a synergistic interaction of core motif sequences in the promoter or region upstream to the promoter of zein genes with various types of transcription factors that interact either directly with the promoter's core sequences or indirectly. In cereals, one of the interesting features of genes encoding prolamines is the existence of a well-known P-box cis-regulatory element, commonly found in the promoter. This P-box or prolamine box element is located $300 \mathrm{bp}$ upstream of the translation start site (TSS). One of the most prominent features of P-box is the existence of a conserved endosperm-specific TGTAAAG motif (Xu \& Messing 2009a, b). The P-box was shown to bind and interact with PBF transcription factor to mediate zein expression regulation. PBF or Prolamine-box Binding Factor belongs to a special class of proteins, commonly known as Dof proteins, which specifically interact and bind to TGTAAAG sequence motif in P-box. Such interactions were shown to be responsible for regulation and expression of 27-kDa $\gamma$-zein (Marzabal et al. 2008). Similarly, 22-kD $\alpha$-zeins were also shown to be co-regulated by $P B F$. The role of $P B F$ at molecular level was further validated by using $P B F$ 
knockdown mutants (PbfRNAi). The defective expression of $P B F$ in these mutants produced a much lower level of $22-k D a \alpha$ and 27- kDa $\gamma$ zeins (Zhang, Yang \& Wu 2015).

One of the most well characterized and extensively studied TF is $\mathrm{O} 2$. Mutation in $\mathrm{O} 2$ causes inability to bind and interact properly with zein genes in 02 mutant, thereby reducing zein content in the mutant by $50-70 \%$. Zeins are naturally deficient of lysine. Loss of function of $\mathrm{O} 2$, therefore, ultimately leads to an increased accumulation of lysine-rich non zein protein in 02 endosperm. This knowledge later became a core principal to develop QPM varieties (Holding 2014; Mertz Bates \& Nelson 1964; Tsai, Larkins \& Glover 1978). O2 belongs to bZIPtype of TF family and specifically expresses 6 days postpollination in the developing endosperm ( $\mathrm{Li}$ et al. 2014; Zhang et al. 2018). O2 targets a specific cis-regulatory conserved motif, referred to as $\mathrm{O} 2$ box, located upstream of the promoters of 22- and 19- kDa $\alpha$-zein genes and mediate their expression directly. Two recent studies demonstrated that the transcription of all zein genes, except for the $16-\mathrm{kD} \gamma$-zein gene, is affected by the $o 2 \mathrm{mu}$ tation. Since, $\mathrm{O} 2$ is dysfunctional in $o 2$ mutant, it has been demonstrated recently that the mutant allele of $\mathrm{O} 2$ affect the regulation of transcription and gene expression of all types of zein genes. The only exception in this case is $16-\mathrm{kD} \gamma$-zein gene where no direct or indirect regulation of expression by $o 2$ has been validated so far ( $\mathrm{Li}$ et al. 2015a, b; Zhang et al. 2016). $\alpha$-zeins are the direct regulatory targets in 02 mutant, where the loss of function mutation in $\mathrm{O} 2 \mathrm{TF}$ down regulates the transcription level of both 19 - and $22-\mathrm{kDa}$ zeins; the later one is down-regulated at much higher level than the former one. $\mathrm{O} 2$ transcriptionally regulates a diverse range of targets which include both zein and non-zein genes. The most commonly investigated three non zein targets of O2 include lactoglutathione lysase, pyruvate orthophosphate dikinase and taxilin ( $\mathrm{Li}$ et al. 2015a, b). Cointeraction of Taxilin and $\mathrm{O} 2$ leads to a cascade of reactions which change the subcellular localization of $\mathrm{O} 2$ protein, and ultimately affect the regulation of zein genes (Zhang et al. 2012). Contrary to $O 2$ and PBF, which express specifically in endosperm after pollination, the products of all other genes show ubiquitous expression in different tissues throughout the plant developmental phases (Zhang et al. 2018). O2 not only regulates genes at transcriptional or translational level, but also mediate modifications at epigenetic level. The interaction of chromatin modifiers with $\mathrm{O} 2$ involves changes in histone proteins and DNA methylation status and, therefore, activates zein genes (Locatelli, Piatti, Motto \& Rossi 2009).

Besides classical TFs-encoding genes $O 2$ and $P B F$, some other transcriptional factors have been identified and characterized to elucidate their regulatory role in the expression of zein genes. For example, MADS-box protein TF, namely ZmMADS47, was shown to interact with $O 2$. ZmMADS47 regulates the expression of $\alpha$ - and $50-\mathrm{kDa} \gamma$ - zeins by binding to CATGT conserved motif in the promoters of these genes. The down regulation of these two genes by ZmMADS47 in RNAi lines affects the normal development and produces low size PBs. However, ZmMADS47 alone is incapable of activating the transcription of zein genes. Instead, it recruits $\mathrm{O} 2$ to trans-activate the promoters and mediate zein genes expression (Qiao et al. 2016). Similarly, two other transcriptional factors $O H P 1$ and $O H P 2$, were shown to recognize and trans-activate all of the $\alpha$-zein promoters. However, the effect of transactivation mediated by $\mathrm{O} 2$ alone has been found much higher than OHP1 and $\mathrm{OHP} 2$ did. Both $\mathrm{O} 2$ and $\mathrm{OHPs}$ are the products of an ancient duplication event. However, both of them diverged significantly and acquired specific functions; $\mathrm{O} 2$ now acts as major while $O H P 1$ and $O H P 2$ as minor transcription factors (Yang, Ji \& Wu 2016). More recently, a newly identified TF, ZmbZIP22, has been found to target ACAGCT $\mathrm{CA}$ element in the promoter of $27-\mathrm{kDa} \gamma$-zein and activate the expression of this genes in transiently transformed tobacco leaves. The CRISPR/Cas9-generated zmbzip22 knockdown mutants significantly reduced the transcript level of $27-\mathrm{kDa} \gamma$-zein gene. Besides interacting in-vivo with $27-\mathrm{kD} \gamma$-zein gene, ZmbZIP22 additionally targets OHPS, $O 2$ and $P B F$, thus synergistically mediate the expression of 27-kD $\gamma$-zein (Li et al. 2018, b). More recently, two new transcription factors ZmNAC128 and $Z m N A C 130$ were shown to have regulatory functions, coordinating the accumulation of starch and proteins at filling stage from 10 to 35 days post pollination through the transcriptional regulation of the $16-\mathrm{kDa} \gamma$-zein genes and at least Bt2 (Zhang, Dong, Ji, Wu \& Messing 2019). The current advances in genome sequencing technologies and availability of high quality reference genome of maize lines will further assist in hunting for more TFs that could possibly shed light on the molecular mechanism underlying zein gene expression and regulation.

\section{The down-stream role of zein in seed formation}

The storage proteins of cereals, such as zeins of maize, play a central role to determine the final nutritional quality of maize grain. The nutritional quality of kernel is a trait of prime importance for human diets not only in developed world, but also in developing countries where people are suffering from malnutrition. Majority of cereals are consumed as processed entities. Thus, protein contents of a kernel are of critical importance in this context because they equally affect the physical and functional features of kernels during and after their processing. In maize seed, zein protein contents make a large bulk of the stored proteins; accounting for $70 \%$ of 
the total $10 \%$ stored proteins (Flint-Garcia et al. 2009). Thus, the downstream role of zein proteins alone and in combination with starch is vital for the proper seed development. In a wild-type maize kernel, the inner and central part of the endosperm is starchy, which do not allow the light to pass through and turns opaque. In contrast, peripheral part of the endosperm easily permits the light to pass through and turns vitreous (Gibbon \& Larkins 2005). Evidence from of previous studies suggests that the development of opaque endosperm is the interactive outcome of PBs, cytoplasm and starch granules (Duvick 1961; Zhang et al. 2018). The molecular mechanism responsible for opaque endosperm formation during seed maturation is poorly understood. It has been inferred from available data that interaction of nonzeins with zein proteins and their final relative accumulation inside PBs are instrumental to the final architecture of maize opaque endosperm. Different members of zein proteins are sequestered into the PBs which in turn surrounds the starch granules in the cytoplasm to give proper architecture to the kernel during seed formation. However, the relative amount of zeins proteins is much higher in peripheral part of the kernel as compared to central opaque region. Moreover, abiotic stress, such as nitrogen depletion that cause reduced zein synthesis may lead to seed endosperm with high starch contents and soft texture (Holding 2014). At seed maturity, cell desiccation and hypersensitive response (HR) lead to the degradation of the endomembrane system. Consequently, the peripheral part of the developing endosperm accumulates starch particles along with $\beta$ and $\gamma$ zein-rich PBs inside a scaffoldlike structure. During seed maturation, the central part of endosperm retains starch granules and turns soft while the peripheral region become vitreous and hard when the aforementioned components get condensed (Gibbon et al. 2003). In $o 2$ mutant, the tiny size and drastically low number of PBs along with naked starch particles embedded in endosperm ultimately result in an opaque and starchy phenotype (Wolf, Khoo \& Seckinger 1967).

Majority of zein proteins stored in maize kernel belong to $\alpha$-zein family where they contribute to the kernel overall hardness and sulfur storage (Wu et al. 2012). The process of $\mathrm{PBs}$ biogenesis is a gradual process, which starts from outer part to the inner part and involves the sequestering of various types of zein inside PBs. $\beta$ - and $\gamma$-zein, residing at the outer surface of PBs, facilitate the sequestration of $\alpha$ - and $\delta$-zeins into the lumen of PBs, while $\gamma$-zeins insure the overall stability of PBs during seed maturation (Holding 2014). The whole process of PBs development is precisely regulated at multiple levels. Such precise check and control system include interaction and spatial-temporal regulation of RNA transcription of various types of zein genes (Kim et al. 2002; Woo, Hu, Larkins \& Jung 2001). The mechanism of high level of zeins accumulation and packaging in the PBs, and their ultimate effect on the development of kernel is a complicated process. Zein proteins are distributed as a discrete layer of tiny particles over the surface of ER. Among all four classes of zein genes, $\gamma$-zein genes are the one which show the earliest expression at the time of PBs initiation. This could be the most likely explanation as why PBs entirely consists of $\gamma$-zein at the start of PBs biogenesis (Woo et al. 2001). As soon as PBs expand, the $\delta$ - and $\alpha$ - zeins are accumulated in the lumen of PBs, where $\gamma$-zeins finally form a capsule-type protein core around them. The final symmetry of PBs consists of a junction layer of $22-k D a \alpha$-zein between the peripheral and central layers of $\gamma$-zeins and $19-\mathrm{kDa}$ - $\alpha$-zeins, respectively (Holding et al. 2007). Selective downregulation has shown that $\gamma$-zeins have specialized roles in kernel development albeit functionally redundant (Guo et al. 2013).

\section{The novel methods applying in zein studies}

With the advancement of modern biotechnological and genome editing technologies, the structure and function of various types of zein genes have been investigated and different components regulating maize endosperm have been characterized. The modern methods applied in zein research include transgenic overexpression, knockdown by RNA interference, deletion mutagenesis and genome editing tools, for example CRISPR/Cas9. Most of the studies have focused on using these tools to elucidate the role of zein genes in maize endosperm development. For instance, the heterologous expression of $5^{\prime}$ UTR along with promoter of $27-\mathrm{kDa}$ and CDS of $10-\mathrm{kD} \delta$-zeins enhanced the overall methionine level in transgenic maize lines. The transgenic lines showed a stable expression of the transgene and retained the expected levels of methionine for several backcross generation. Transgenic methionine supplemented poultry diet showed satisfactory results, implying that transgenic maize was a suitable alternative of free methionine-added diet (Lai \& Messing 2002). High lysine contents of the maize endosperm contribute to better nutritional quality. In order to increase the lysine contents in the mature kernel, the transgenes must be under tight control so as to avoid the interactions of transgenic proteins with the machinery of programmed cell death. Moreover, the transgene must be driven by a strong promoter to express highly and retain high level of transgenic protein in endosperm (Holding 2014). For this purpose, strategies have been designed to express lysine-rich, nonmaize proteins specifically in endosperm. One such study has documented the expression of lysine-rich non-maize protein under the control of $\gamma$ - or $\alpha$-zein promoters to elevate the transgenic lysine contents in maize (Kriz 2009). An alternative strategy for increasing the lysine contents, specifically in endosperm, is to modify the CDS of zein 
genes per se. Using this transgenic strategy, lysine residues were modified in $19-\mathrm{kD} \alpha$-zein gene and expressed in Xenopus oocytes, which accumulated respective modified protein in PBs-like structures (Holding et al. 2007). It has been investigated that substitution of a fraction of native zein proteins with a modified protein significantly affects the kernel protein quality. Besides $\alpha$-zein family modification, 27-kDa $\gamma$-zein has been subjected to modification of lysine residues because it is not only the major $o 2$ modifier but also an initiator of PBs formation, as well as accumulates abundantly in the endosperm (Holding 2014; Liu et al. 2016).Certain residues in $27-\mathrm{kDa} \gamma$-zein were modified by substituting Pro-Xaa region with proline-lysine sequences. The modified $27-\mathrm{kDa} \gamma$-zein was transiently expressed in maize, where the respective modified proteins showed co-localization with endogenous $\gamma$ - and $\alpha$ zein proteins (Torrent et al. 1997).

In wild type maize, the accumulation of $\alpha$-zeins in high amount negatively affects the nutritional quality, thereby reducing the lysine-rich proteins in endosperm. Thus, it is mandatory to design a strategy which can down-regulate the contents of $\alpha$-zeins, and in return elevate the level of transgenic proteins enriched in lysine. RNA interference (RNAi) provides an effective tool to knock out or knock down the zein genes for endosperm modification (Holding \& Messing 2013; Liu et al. 2016). The RNAi constructs of $22-\mathrm{kDa} \alpha$-zein were expressed in maize and the transgenic lines exhibited a considerable reduction in the 22-kD $\alpha$-zein and elevated level of lysine contents. Accumulation of low levels of $22-\mathrm{kDa} \alpha-$ zein causes deformities in the structure and size of PBs (Segal, Song \& Messing 2003), suggesting that the possible role of $22-k D a \alpha$-zein to correctly package $19-k D a$ $\alpha$-zein inside PB. This role is further supported by the existence of $22-\mathrm{kDa} \alpha$-zein as an outer layer, surrounding the inner layer of $19-\mathrm{kDa} \alpha$-zein during PBs development (Holding et al. 2007). However, when both 19- and $22-\mathrm{kDa} \alpha$-zeins were silenced, no observable effects on the number and shape of PBs were found, implying that $\alpha$-zeins are not necessary for PBs, though mandatory for PBs filling (Guo et al. 2013; Huang, Frizzi, Florida, Kruger \& Luethy 2006; Wu \& Messing 2011). Besides $\alpha$-zein family, RNAi was used to get insight into the functional role of $\gamma$-zeins family. The study took advantage of the high sequence similarity of 16 - and $27 \mathrm{kDa} \gamma$-zeins as well as $15-\mathrm{kDa} \beta$-zein to knock them down, respectively. However, the silencing of these genes has a minor effect on the shape, size and number of PBs (Wu \& Messing 2010). Several studies have unraveled the possible link of $27-\mathrm{kDa} \gamma$-zein high expression with endosperm development in QPM lines. However, the mechanism underlying the QPM endosperm modification by $27-\mathrm{kDa} \gamma$-zein remained elusive until recently when the QTL for $27-\mathrm{kDa}$ $\gamma$-zein was cloned and functionally validated by RNAi (Liu et al. 2016; Wu \& Messing 2010; Yuan et al. 2014). Contrary to $22-\mathrm{kDa} \alpha$ - zein genes, no significant expression variations were observed in the level of $27-\mathrm{kDa} \gamma$-zein gene in $o 2$ mutant. In addition, it was also subject to transcriptional regulation by $P B F$ and $O H P S$ in $o 2$ mutant (Zhang et al. 2018). The knock down of $P B F$ in transgenic RNAi maize (PbfRNAi) produced significantly low level of 27-kDa $\gamma$-zein proteins (Wu \& Messing 2012). When PbfRNAi/+;o2 line was crossed with K0326Y QPM, a stable inheritance of transgene was observed in about $50 \%$ of the progenies. The presence of low level of $27-\mathrm{kDa} \gamma$ zein and opaque endosperm in K0326Y $\times 02$; PbfRNAi/+ transgenic lines suggest that $P B F$ down regulates the transcription of 27-kD $\gamma$-zein (Liu et al. 2016). Besides directly targeting zein genes, RNAi was used to silence transcription factor ZmMADS47. The RNAi lines of ZmMADS47 exhibited down regulation of the expression of $50-\mathrm{kDa} \gamma$ - and $\alpha$ zein genes, and their PBs quite smaller in size (Qiao et al. 2016).

While RNAi transgenes and other different kinds of mutations cause partial expression, deletion gene mutagenesis leads to complete loss of function phenotypes. Radiations are the most potent agents to carry out random large-insert deletion mutagenesis. The deletion of Opm loci in QPM lines, when targeted with $\gamma$-radiations, produced a variety of $\alpha$ - and $\gamma$-zein null mutants in a pool of $\sim 300 \mathrm{M} 3$ families (Holding 2014). Of this M3 family, two of the striking null mutants were mutant lines 107 and mutant line 198. The former one is complete loss of function mutant containing undetectable amount of 50- and 27- $\gamma$-zeins, while the later one exhibited reduced amount of 19 - and 22-kDa $\alpha$-zeins. The results of this study suggest that the large insert targeted by $\gamma$-radiations in the respective QPM line 108 might contain both 27- and 50- $\gamma$-zein genes because they are located at very close genetic distance on the same chromosome. NGS data further validated that more than $1 \mathrm{Mb}$ large insert deleted by $\gamma$-radiations contained both 27- and 50- $\gamma$-zein genes (Holding 2014; Holding et al. 2008; Yuan et al. 2014). Though RNAi transgene constructs and deletion mutagenesis have been proved to be effective tools in elucidating structural and functional roles of different members of zein family, their mutation effect is not precise but random across the genome. The advent of novel genome editing technologies, especially CRISPR/Cas9, holds great promise for specifically mutating the zein genes per se as well as those participating in the regulation of maize endosperm development. One such study has recently used CRISPR/Cas9 technology to target transcription factor, namely ZmbZIP22, which specifically express in maize endosperm. CRISPR/Cas9-editted mutant plants (zmbzip22) accumulated reduced amount of $27-\mathrm{kD} \gamma$-zein, demonstrating ZmbZIP22-mediated regulation of the 
expression of 27-kD $\gamma$-zein gene (Li et al. 2018, b). Sorghum has a similar protein composition as maize. Similar to maize zeins, the major storage proteins in sorghum are kafirins. Kafirins are also devoid of lysine and tryptophan and show poor digestibility. These properties of kafirin lead to serious malnutrition problems when sorghum is consumed as a major protein source. The investigation of a sorghum mutant exhibiting elevated lysine and betterprotein digestibility was shown to be associated with a point mutation in $22 \mathrm{kDa}$ alpha kafirin, similar to $f l 2 \mathrm{mu}-$ tant of maize. This mutant was later used to develop sorghum varieties with easily digestible kafirins and increased lysine contents (Wu, Yuan, Guo, Holding \& Messing 2013). A multi-genic family, namely $k 1 C$, encodes kafirins proteins in sorghum. Similar to maize, CRISPR-Cas9 approach was successfully used to edit $k 1 C$ genes. The resultant CRISPR-mutants sorghum lines produced low level of kafirins, better digestibility and improved protein quality (Li et al. 2018, b).

\section{Concluding remarks}

In the past 50 years, much progress has been made to investigate the structural, functional and molecular basis of zein gene family, both in maize and its paralogues in other cereals. The core objective was to decipher the mechanism underlying the endosperm development for better nutritional quality (Xu \& Messing 2009a, b). The information obtained from the past decades research contributed to the identification and molecular characterization of more than dozens of maize opaque mutants, development of maize varieties with modified endosperm via traditional breeding and modern biotechnological techniques and elucidation of transcriptional regulatory networks mediating zein family expression and regulation. One of the promising research paradigms in the coming days is foreseen to characterize more and more maize opaque mutants and to identify their respective cis and trans regulatory pairs in gene networks analysis. Such studies will ultimately resolve the complex mechanism governing the maize endosperm development. Moreover, the applications of latest genome editing tools, especially CRISPR/Cas9 technology, will further unravel the complexity of zein-gene family and improve our understanding about the genetic and regulatory factors mediating kernel development for better nutritional quality. The recent advances in next generation sequencing technologies, such as economically cost-effective and error free sequencing and assembling will further revolutionize the zein gene research. The availability of high quality reference genome of B73 (Jiao et al. 2017) and the completion of ongoing genome sequencing of more maize lines will greatly contribute to the investigation of zein family across different maize genetic backgrounds in the near future.

\section{Abbreviations}

bZIP: Basic Leucine Zipper domain; CRISPR: Clustered Regularly Interspaced Short Palindromic Repeats; MGD1: Mono Galactosyl Diacylglycerol synthase1; 02: Opaque Mutant2; OHPs: O2 Heterodimerizing Proteins; Opm: Opaque2 Modifiers; P-box: Prolamin-box; PBs: Protein Bodies; QPM: Quality Protein Maize; QTLs: Quantitative Trait Loci; SDS-gel: Sodium Dodecyl Sulfate-gel

\section{Acknowledgments \\ The first author acknowledges the financial support from China Post- Doctoral Council and Jiangsu Academy of Agricultural Sciences for Post- doctoral program.}

\section{Authors' contributions}

NUK and ZH conceived the idea, draft and wrote the manuscript. MS, NL and MMUK contributed to the drafting of the manuscript. All authors read and approved the final manuscript.

\section{Funding}

The work was financially supported by Key Research and Development Program of Jiangsu Province (BE2017365). It is declared that the funding body had no role in the design of the study, analysis, or interpretation of data, or in writing the manuscript.

\section{Availability of data and materials}

Data sharing is not applicable to this article as no datasets were generated or analyzed during the current study.

Ethics approval and consent to participate

Not applicable.

Consent for publication

Not applicable.

\section{Competing interests}

The authors declare that they have no competing interests.

\section{Author details}

${ }^{1}$ Institute of Crop Germplasm and Biotechnology, Provincial Key Laboratory of Agrobiology, Jiangsu Academy of Agricultural Sciences, Nanjing 210014, China. ${ }^{2}$ Department of Agronomy, Faculty of Agriculture, Mansoura

University, Mansoura, Egypt. ${ }^{3}$ Faculty of Agriculture, Gomal University, Dera Ismail Khan, KP, Pakistan.

Received: 8 April 2019 Accepted: 30 October 2019

Published online: 23 December 2019

\section{References}

Akalu, G., Taffesse, S., Gunaratna, N. S., \& De Groote, H. (2010). The effectiveness of quality protein maize in improving the nutritional status of young children in the Ethiopian highlands. Food and Nutrition Bulletin, 31, 418-430.

Babu, B. K., Agrawal, P. K., Saha, S., \& Gupta, H. S. (2015). Mapping QTLs for opaque2 modifiers influencing the tryptophan content in quality protein maize using genomic and candidate gene-based SSRs of lysine and tryptophan metabolic pathway. Plant Cell Reports, 34, 37-45.

Chen, J., Zeng, B., Zhang, M., Xie, S., Wang, G., et al. (2014). Dynamic transcriptome landscape of maize embryo and endosperm development. Plant Physiology, 166, 252-264.

Coleman, C. E., Clore, A. M., Ranch, J. P., Higgins, R., Lopes, M. A., \& Larkins, B. A. (1997). Expression of a mutant alpha-zein creates the floury2 phenotype in transgenic maize. Proceedings of the National Academy of Sciences of the United States of America, 94, 7094-7097.

Das, O. P., Levi-Minzi, S., Koury, M., Benner, M., \& Messing, J. (1990). A somatic gene rearrangement contributing to genetic diversity in maize. Proceedings of the National Academy of Sciences of the United States of America, 87, 78097813.

Dong, J. Q., Feng, Y. P., Kumar, D., Zhang, W., Zhu, T. T., Luo, M. C., \& Messing, J. (2016). Analysis of tandem gene copies in maize chromosomal regions reconstructed from long sequence reads. Proceedings of the National Academy of Sciences of the United States of America, 113, 7949-7956.

Duvick, D. N. (1961). Protein granules of maize endosperm cells. Cereal Chemistry, $38,374-385$. 
Feng, F., Qi, W. W., LV, Y., Yan, S., Xu, L., Yang, W., Yuan, Y., Chen, Y. H., Zhao, H., \& Song, R. T. (2018). OPAQUE11 is a central hub of the regulatory network for maize endosperm development and nutrient metabolism. The Plant Cell, 30, 375-396.

Feng, L., Zhu, J., Wang, G., Tang, Y., Chen, H., Jin, W., Wang, F., Mei, B., Xu, Z., \& Song, R. (2009). Expressional profiling study revealed unique expressional patterns and dramatic expressional divergence of maize alpha-zein super gene family. Plant Molecular Biology, 69, 649.

Flint-Garcia, S. A., Bodnar, A. L., \& Scott, M. P. (2009). Wide variability in kernel composition, seed characteristics, and zein profiles among diverse maize inbreds, landraces, and teosinte. Theoretical and Applied Genetics, 119, 1129-1142.

Geetha, K. B., Lending, C. R., Lopes, M. A., Wallace, J. C., \& Larkins, B. A. (1991). Opaque-2 modifiers increase gamma-zein synthesis and alter its spatialdistribution in maize endosperm. The Plant Cell, 3, 1207-1219.

Gibbon, B. C., Wang, X., Larkins, B. A. (2003). Altered starch structure is associated with endosperm modification in Quality Protein Maize. Proceedings of the National Academy of Sciences of the United States of America, 100, 15329-15334.

Gibbon, B. C., \& Larkins, B. A. (2005). Molecular genetic approaches to developing quality protein maize. Trends in Genetics, 21, 227-233.

Gunaratna, N. S., De Groote, H., Nestel, P., Pixley, K. V., \& McCabe, C. P. (2010). A meta-analysis of community-based studies on quality protein maize. Food Policy, 35, 202-210.

Guo, X., Yuan, L., Chen, H., Sato, S. J., Clemente, T. E., \& Holding, D. R. (2013). Nonredundant function of zeins and their correct stoichiometric ratio drive protein body formation in maize endosperm. Plant Physiology, 162, 1359-1369.

Holding, D. R. (2014). Recent advances in the study of prolamine storage protein organization and function. Frontiers in Plant Science, 5, 276.

Holding, D. R., Hunter, B. G., Chung, T. J., Gibbon, B. C., Ford, C. F., Bharti, A. K., Messing, J., Hamaker, B. R., \& Larkins, B. A. (2008). Genetic analysis of opaque2 modifier loci in quality protein maize. Theoretical and Applied Genetics, 117, 157-170.

Holding, D. R., Hunter, B. G., Klingler, J. P., Wu, S., Guo, X. M., Gibbon, B. C., Wu, R. L., Schulze, J. M., Jung, R., \& Larkin, B. A. (2011). Characterization of opaque2 modifier QTLs and candidate genes in recombinant inbred lines derived from the K0326Y quality protein maize inbred. Theoretical and Applied Genetics, 122, 783-794.

Holding, D. R., Meeley, R. B., Hazebroek, J., Selinger, D., Gruis, F., Jung, R., et al. (2010). Identification and characterization of the maize arogenate dehydrogenase gene family. Journal of Experimental Botany, 61, 3663-3673.

Holding, D. R., \& Messing, J. (2013). "Evolution, structure, and function of prolamin storage proteins", in Seed Genomics, ed. P. Becraft (New York: John Wiley \& Sons), 139-158.

Holding, D. R., Otegui, M. S., Li, B. L., Meeley, R. B., Dam, T., Hunter, B. G., et al. (2007). The maize floury1 gene encodes a novel endoplasmic reticulum protein involved in zein protein body formation. The Plant Cell, 19, 2569-2582

Huang, S., Frizzi, A., Florida, C. A., Kruger, D. E., \& Luethy, M. H. (2006). High lysine and high tryptophan transgenic maize resulting from the reduction of both 19- and 22-kD alpha-zeins. Plant Molecular Biology, 61, 525-535.

Jiao, Y. P., Peluso, P., Shi, J. H., Liang, T., Stitzer, M. C., Wang, B., Campbell, M. S., et al. (2017). Improved maize reference genome with single-molecule technologies. Nature. https://doi.org/10.1038/nature22971.

Kellogg, E. A. (2001). Evolutionary history of the grasses. Plant Physiology, 125, 1198-1205.

Kim, C. S., Gibbon, B. C., Gillikin, J. W., Larkins, B. A., Boston, R. S., \& Jung, R. (2006). The maize Mucronate mutation is a deletion in the $16-\mathrm{kDa}$ gamma-zein gene that induces the unfolded protein response. The Plant Journal, 48, 440-451.

Kim, C. S., Hunter, B. G., Kraft, J., Boston, R. S., Yans, S., Jung, R., \& Larkins, B. A. (2004). A defective signal peptide in a 19-kD alpha-zein protein causes the unfolded protein response and an opaque endosperm phenotype in the maize De*-B30 mutant. Plant Physiology, 134, 380-387.

Kim, C. S., Woo, Y. M., Clore, A. M., Burnett, R. J., Carneiro, N. P., \& Larkins, B. A. (2002). Zein protein interactions, rather than the asymmetric distribution of zein mRNAs on endoplasmic reticulum membranes, influence protein body formation in maize endosperm. The Plant Cell, 14,655-672.

Kriz, A. L. (2009). Enhancement of amino acid availability in corn grain. In A. L. Kriz \& B. A. Larkins (Eds.), Molecular genetic approaches to maize improvement (pp. 79-89). Heidelberg: Springer. https://doi.org/10.1007/978-3-540-68922-5.

Lai, J. S., and Messing, J. (2002). Increasing maize seed methionine by mRNA stability. Plant Journal, 30, 395-402.
Li, A., Jia, S., Yobi, A., Ge, Z., Sato, S. J., Zhang, C., Angelovici, R., Clemente, T. E., \& Holding, D. R. (2018). Editing of an alpha-Kafirin gene family increases, digestibility and protein quality in Sorghum. Plant Physiology, 177, 1425-1438.

Li, C., Qiao, Z., Qi, W., Wang, Q., Yuan, Y., et al. (2015a). Genome-wide characterization of cis-acting DNA targets reveals the transcriptional regulatory framework of opaque2 in maize. The Plant Cell, 27, 532-545.

Li, C. B., Yue, Y. H., Chen, H. J., Qi, W. W., \& Song, R. T. (2018). The ZmbZIP22 transcription factor regulates $27-k D \gamma$-Zein gene transcription during maize endosperm development. The Plant Cell. https://doi.org/10.1105/tpc.18.00422.

Li, G. S., Wang, D. F., Yang, R. L., Logan, K., Chen, H., Zhang, S. S., Skaggs, M. I., et al. (2014). Temporal patterns of gene expression in developing maize endosperm identified through transcriptome sequencing. Proceedings of the National Academy of Sciences of the United States of America, 111, 7582-7587.

Li, Q., Wang, J., Ye, J., Zheng, X., Xiang, X., Li, C., Fu, M., Wang, Q., Zhang, Z., \& Wu, $Y$. (2017). The maize imprinted gene Floury3 encodes a PLATZ protein required for tRNA and $5 \mathrm{~S}$ rRNA transcription through interaction with RNA polymerase III. The Plant Cell, 29, 2661-2675.

Liu, C. N., \& Rubenstein, I. (1993). Transcriptional characterization of an alpha-zein gene cluster in maize. Plant Molecular Biology, 22, 323-336.

Liu, H., Shi, J., Sun, C., Gong, H., Fan, X., Qiu, F., Huang, X., Feng, Q. et al. (2016). Gene duplication confers enhanced expression of 27-kDa gamma-zein for endosperm modification in quality protein maize. Proceedings of the National Academy of Sciences of the United States of America, 113, 4964-4969.

Locatelli, S., Piatti, P., Motto, M., \& Rossi, V. (2009). Chromatin and DNA modifications in the Opaque2-mediated regulation of gene transcription during maize endosperm development. The Plant Cell, 21, 1410-1427.

Lopes, M. A., \& Larkins, B. A. (1991). Gamma-zein content is related to endosperm modification in quality protein maize. Crop Science, 31, 1655-1662.

Marzabal, P., Gas, E., Fontanet, P., Vicente-Carbajosa, J., Torrent, M., et al. (2008). The maize Dof protein PBF activates transcription of gamma-zein during maize seed development. Plant Molecular Biology, 67, 441-454.

Mertz, E. T., Bates, L. S., \& Nelson, O. E. (1964). Mutant gene that changes protein composition and increases lysine content of maize endosperm. Science., 145, 279-280.

Miclaus, M., Xu, J. H., \& Messing, J. (2011). Differential gene expression and Epiregulation of alpha Zein gene copies in maize haplotypes. PLoS Genetics, 7(6), e1002131.

Myers, A. M., James, M. G., Lin, Q., Yi, G., Stinard, P. S., Hennen-Bierwagen, T. A., \& Becraft, P. W. (2011). Maize opaque5 encodes monogalactosyldiacylglycerol synthase and specifically affects galactolipids necessary for amyloplast and chloroplast function. The Plant Cell, 23, 2331-2347.

Nuss, E. T., \& Tanumihardjo, S. A. (2011). Quality protein maize for Africa: Closing the protein inadequacy gap in vulnerable populations. Advances in Nutrition, 2, 217-224.

Osborne, T. B., \& Mendel, L. B. (1914). Nutritional properties of proteins of the maize kernel. The Journal of Biological Chemistry, 18, 1-16.

Planta, J., Xiang, X., Leustek, T., \& Messing, J. (2017). Engineering sulfur storage in maize seed proteins without apparent yield loss. Proceedings of the National Academy of Sciences of the United States of America, 114, 11386-11391.

Qiao, Z., Qi, W. W., Wang, Q., Feng, Y., Yang, Q., Zhang, N., Wang, S. S., Tang, Y. P., \& Song, R. T. (2016). ZmMADS47 regulates zein gene transcription through interaction with Opaque2. PLoS Genetics, 12, e1005991.

Ren, Y., Yobi, A., Marshall, L., Angelovici, R., Rodriguez, O., \& Holding, D. R. (2018). Generation and evaluation of modified Opaque-2 popcorn suggests a route to quality protein popcorn. Frontiers in Plant Science. https://doi.org/10.3389/ fpls.2018.01803.

Rolfes, S. R., Pinna, K., \& Whitney, E. (2009). Protein: amino acids. In: Understanding normal and clinical nutrition (p. 198). Belmont: Wadsworth.

Schmidt, R. J., Ketudat, M., Aukerman, M. J., \& Hoschek, G. (1992). Opaque-2 is a transcriptional activator that recognizes a specifc target site in $22-\mathrm{kD}$ zein genes. The Plant Cell, 4, 689-700.

Segal, G., Song, R. T., \& Messing, J. (2003). A new opaque variant of maize by a single dominant RNA-interference-inducing transgene. Genetics., 165, 387-397.

Song, R., Llaca, V., Linton, E., \& Messing, J. (2001). Sequence, regulation, and evolutionof the maize 22-kD alpha zein gene family. Genome Research, 11, 1817-1825.

Song, R., \& Messing, J. (2002). Contiguous genomic DNA sequence comprising the 19-kD Zein gene family from maize. Plant Physiology, 130, 1626-1635.

Song, R., \& Messing, J. (2003). Gene expression of a gene family in maize based on non-collinear haplotypes. Proceedings of the National Academy of Sciences of the United States of America, 100, 9055-9060. 
Swigonova, Z., Swigoňová, Z., Lai, J. S., Ma, J. X., Ramakrishna, W., Llaca, V., Jeffrey, L., Bennetzen, J. L., \& Messing, J. (2004). Close split of sorghum and maize genome progenitors. Genome Research, 14, 1916-1923.

Torrent M., Alvarez I., Geli, M.I., Dalcol, i., Ludevid D. (1997). Lysine-rich modified Yzeins accumulate in protein bodies of transiently transformed maize endosperms. Plant Molecular Biology, 34, 139-149.

Tsai, C. Y., Larkins, B. A., \& Glover, D. V. (1978). Lnteraction of the opaque-2 gene with starch-forming mutant genes on the synthesis of zein in maize endosperm. Biochemical Genetics, 16, 883-896.

Van Hoof, A., \& Green, P. J. (1996). Premature nonsense codons decrease the stability of phytohemagglutinin mRNA in a position-dependent manner. The Plant Journal, 10, 415-424.

Wang, G., Qi, W. W., Wu, Q., Yao, D. S., Zhang, J. S., Zhu, J., Wang, G., Wang, G. F., Tang, Y. P., \& Song, R. T. (2014). Identification and characterization of maize floury4 as a novel semidominant opaque mutant that disrupts protein body assembly. Plant Physiology, 165, 582-594.

Wang, G., Zhang, J., Wang, G., Fan, X., Sun, X., Qin, H., Xu, N., Zhong, M., Qiao, Z., Tang, Y., \& Song, R. T. (2014). Proline responding1 plays a critical role in regulating general protein synthesis and the cell cycle in maize. The Plant Cell, 26, 2582-2600.

Wang, G. F., Wang, F., Wang, G., Wang, F., Zhang, X. W., Zhong, M. Y., Zhang, J., Lin, D. B., Tang, Y. P., Xu, Z. K., \& Song, R. T. (2012). Opaque1 encodes a myosin $\mathrm{XI}$ motor protein that is required for endoplasmic reticulum motility and protein body formation in maize endosperm. The Plant Cell, 24, 3447-3462.

Wheeler, T., \& von Braun, J. (2013). Climate change impacts on global food security. Science., 341, 508-513.

Wolf, M. J., Khoo, U., \& Seckinger, H. L. (1967). Subcellular structure of endosperm protein in high-lysine and normal corn. Science., 157, 556-557.

Woo, Y. M., Hu, D. W. N., Larkins, B. A., \& Jung, R. (2001). Genomics analysis of genes expressed in maize endosperm identifies novel seed proteins and clarifies patterns of zein gene expression. The Plant Cell, 13, 2297-2317.

Wu, Y., Holding, D. R., \& Messing, J. (2010). Gamma-zeins are essential for endosperm modification in quality protein maize. Proceedings of the National Academy of Sciences of the United States of America, 107, 12810-12815.

Wu, Y., \& Messing, J. (2012). Rapid divergence of prolamin gene promoters of maize after gene amplification and dispersal. Genetics., 192, 507-519.

Wu, Y., \& Messing, J. (2014). Proteome balancing of the maize seed for higher nutritional value. Frontiers in Plant Science, 5, 240.

Wu, Y., Yuan, L., Guo, X., Holding, D. R., \& Messing, J. (2013). Mutation in the seed storage protein kafirin creates a high-value food trait in sorghum. Nature Communications, 4, 2217.

Wu, Y. R., \& Messing, J. (2011). Novel genetic selection system for quantitative trait loci of quality protein maize. Genetics., 188, 1019-1022.

Wu, Y., and Messing, J. (2010). RNA interference-mediated change in protein body morphology andseed opacity through loss of different zein proteins. Plant Physiology, 153, 337-347.

Wu, Y., Wang, W., and Messing, J. (2012). Balancing of sulfur storage in maize seed. BMC Plant Biology, 12, 77. https://doi.org/10.1186/1471-2229-12-77

$\mathrm{Xu}$, J. H., \& Messing, J. (2009a). Amplification of prolamin storage protein genes in different subfamilies of the Poaceae. Theoretical and Applied Genetics, 119, 1397-1412

Xu, J. H., \& Messing, J. (2009b). Organization of the prolamin gene family provides insight into the evolution of the maize genome and gene duplications in grass species. Proceedings of the National Academy of Sciences of the United States of America, 105, 14330-14335.

Yang, J., Fu, M., Ji, C., Huang, Y., \& Wu, Y. (2018). Maize Oxalyl-CoA Decarboxylase1 degrades oxalate and affects the seed Metabolome and nutritional quality. The Plant Cell, 30, 2447-2462.

Yang, J., Ji, C., \& Wu, Y. R. (2016). Divergent transactivation of maize storage protein Zein genes by the transcription factors Opaque2 and OHPs. Genetics., 204, 581-591.

Yao, D. S., Qi, W. W., Li, X., Yang, Q., Yan, S. M., Ling, H. L., Wang, G., Wang, G. F., \& Song, R. T. (2016). Maize opaque10 encodes a cereal-specifc protein that is essential for the proper distribution of zeins in endosperm protein bodies. PLoS Genetics, 12, e1006270.

Yuan, L., Dou, Y., Kianian, S. F., Zhang, C., \& Holding, D. R. (2014). (2014) deletion mutagenesis identifies a haploinsufficient role for $\gamma$-zein in opaque2 endosperm modification. Plant Physiology, 164, 119-130.
Zhang, N., Qiao, Z., Liang, Z., Mei, B., Xu, Z., \& Song, R. (2012). Zea mays Taxilin protein negatively regulates opaque- 2 transcriptional activity by causing a change in its sub-cellular distribution. PLoS One, 7, e43822.

Zhang, S. S., Zhan, J. P., \& Yadegari, R. (2018). Maize opaque mutants are no longer opaque. Plant Reproduction., 31, 319-326.

Zhang, Z., Dong, J., Ji, C., Wu, Y., \& Messing, J. (2019). NAC-type transcription factors regulate accumulation of starch and protein in maize seeds. Proceedings of the National Academy of Sciences of the United States of America, 116, 11223-11228.

Zhang, Z., Yang, J., \& Wu, Y. (2015). Transcriptional regulation of zein gene expression in maize through the additive and synergistic action of opaque2, Prolamine-box binding factor, and $\mathrm{O} 2$ heterodimerizing proteins. The Plant Cell, 27, 1162-1172

Zhang, Z., Zheng, X., Yang, J., Messing, J., \& Wu, Y. (2016). (2016) maize endosperm specific transcription factors $\mathrm{O} 2$ and PBF network the regulation of protein and starch synthesis. Proceedings of the National Academy of Sciences of the United States of America, 113, 10842-10847.

\section{Publisher's Note}

Springer Nature remains neutral with regard to jurisdictional claims in published maps and institutional affiliations.
Ready to submit your research? Choose BMC and benefit from:

- fast, convenient online submission

- thorough peer review by experienced researchers in your field

- rapid publication on acceptance

- support for research data, including large and complex data types

- gold Open Access which fosters wider collaboration and increased citations

- maximum visibility for your research: over $100 \mathrm{M}$ website views per year

At BMC, research is always in progress.

Learn more biomedcentral.com/submissions 